\title{
Hypothetical Switch of Anti-Vascular Endothelial Growth Factor in Neovascular Age-Related Macular Degeneration: An ARIES Post Hoc Analysis
}

\author{
Cengiz Tuerksever · Gábor Márk Somfai · Susanne Oesch • \\ Tobias Machewitz · Pascal W. Hasler · Sandrine Zweifel
}

Received: November 22, 2021 / Accepted: December 13, 2021 / Published online: January 23, 2022

(C) The Author(s) 2022

\begin{abstract}
Introduction: Switching to an alternative antivascular endothelial growth factor (anti-VEGF) agent has been suggested for patients with neovascular age-related macular degeneration (nAMD) who have a suboptimal response to initial therapy. However, post hoc analyses of some studies have shown that continuation of initial anti-VEGF therapy is, in many cases, associated with stable visual outcomes or gradual gains.
\end{abstract}

Supplementary Information The online version contains supplementary material available at https:// doi.org/10.1007/s40123-021-00448-w.

C. Tuerksever $(\bowtie)$

VISTA Clinic Binningen BL, 4201 Binningen, Switzerland

e-mail: cengiz.tuerksever@vista.ch

G. M. Somfai

Werner H. Spross Foundation for the Advancement of Research and Teaching in Ophthalmology,

Zurich, Switzerland

G. M. Somfai

Department of Ophthalmology, City Hospital Waid and Triemli, Zurich, Switzerland

G. M. Somfai

Department of Ophthalmology, Semmelweis

University, Budapest, Hungary
Methods: This ARIES (ClinicalTrials.gov Identifier: NCT02581891) post hoc analysis describes outcomes in patients with treatment-naïve nAMD receiving treat-and-extend intravitreal aflibercept (IVT-AFL) for 104 weeks, who were identified as meeting criteria for an early hypothetical switch. Patients were categorized retrospectively according to six criteria (presence of central intraretinal and/or subretinal fluid at week 8 or 24 , with/without a next planned treatment interval $\leq 8$ weeks, and with/without gains in best-corrected visual acuity $[\mathrm{BCVA}] \leq 5$ letters [with absolute BCVA $<70$ letters]).

Results: Hypothetical switch criteria were largely met due to the presence of central subretinal fluid rather than intraretinal fluid.

\section{S. Oesch}

Medical Department, Bayer (Schweiz) AG, Zurich, Switzerland

T. Machewitz

Medical Affairs Statistics, Bayer AG, Berlin, Germany

P. W. Hasler

Department of Ophthalmology, University Hospital Basel, Basel, Switzerland

S. Zweifel

Department of Ophthalmology, University Hospital

Zurich, Zurich, Switzerland

S. Zweifel

University of Zurich, Zurich, Switzerland 
Depending on the criterion, $8-46 \%$ of patients were considered to be hypothetical switchers. BCVA outcomes were not worse in the hypothetical switchers, irrespective of criteria. Using criteria of intraretinal/subretinal fluid at week 24 and a next planned treatment interval $\leq 8$ weeks, mean changes in BCVA (letters) from baseline in hypothetical switchers and nonswitchers were: +6.1 (95\% confidence interval [CI] $3.4,8.8)$ and $+6.6(95 \%$ CI $4.7,8.6)$, respectively, at week $24 ;+8.2$ (95\% CI 5.0, $11.3)$ and +7.5 (95\% CI 5.3, 9.7), respectively, at week 52; and $+5.7(95 \%$ CI $1.3,10.1)$ and +3.4 (95\% CI $0.1,6.7)$, respectively, at week 104 .

Conclusions: In newly diagnosed nAMD, there appears little rationale for early switching from IVT-AFL since, with continuous proactive treatment, comparable visual gains can be achieved by patients meeting hypothetical switch criteria compared with those who initially respond well on a treat-and-extend regimen. However, further prospective studies are needed.

Trial registration: ClinicalTrials.gov Identifier: NCT02581891.

Video Summary: Video summary of the ARIES Post Hoc Analysis "Hypothetical Switch of Anti-Vascular Endothelial Growth Factor in Neovascular Age-Related Macular Degeneration” (MP4 54283 KB)

Keywords: Aflibercept; Age-related macular degeneration; Intravitreal injections; Optical coherence tomography; Treat-and-extend; Treatment outcome; Vascular endothelial growth factors

\section{Key Summary Points}

\section{Why carry out this study?}

Several single-arm studies have suggested that there are benefits to switching antivascular endothelial growth factor (antiVEGF) agents in patients with neovascular age-related macular degeneration (nAMD) who have a suboptimal response to treatment of intravitreal anit-VEGF agents; however, without a randomized control group, it is not possible to evaluate the effects of switching treatments without the comparison of continuing the original treatment.

We conducted a post hoc analysis of data from the ARIES intravitreal aflibercept (IVT-AFL) study in which we identified a group of patients as theoretical switchers, i.e., those who did not have have an optimal response to treatment after the first 8 or 24 weeks of treatment. We describe here the functional outcomes of these patients compared with those who did not meet the switch criteria.

\section{What was learned from the study?}

In this post hoc analysis of the ARIES study, for patients who met criteria for a hypothetical switch of anti-VEGF treatment, mean initial improvements in best-corrected visual acuity were maintained or even numerically improved with continued proactive, individualized treat-and-extend IVT-AFL therapy for nAMD.

This post hoc analysis suggests that there appears little rationale for early switching from IVT-AFL in patients with nAMD.

With continuous proactive treatment, comparable visual gains can be achieved by patients meeting hypothetical switch criteria compared with those who initially respond well. 


\section{DIGITAL FEATURES}

This article is published with digital features, including a video abstract, to facilitate understanding of the article. To view digital features for this article, go to https://doi.org/10.6084/ m9.figshare.17185727.

\section{INTRODUCTION}

Neovascular age-related macular degeneration (nAMD) is associated with a deterioration in visual functioning and significant socioeconomic implications through direct and indirect medical costs, including loss of earnings, loss of healthy life, and a clinically relevant reduction in vision-related quality of life [1-3]. The substantial global burden of nAMD is well established, with various models indicating a considerable increase in the number of affected individuals [4-6], largely due to an aging population. Agents targeting vascular endothelial growth factor (VEGF) have had a significant impact on treatment [5]. However, despite their introduction as anti-VEGF agents over 15 years ago [7], scientific debate continues regarding the choice of therapeutic regimens and adequate monitoring requirements [8].

Proactive, individualized treat-and-extend (T\&E) regimens are emerging as the preferred method to maintain the improvements in functional and anatomic outcomes associated with anti-VEGF injections while reducing the monitoring/treatment burden over the medium/long term for patients and healthcare systems [9]. Due to lockdown during the COVID19 pandemic, clinical appointments have been missed and the number of treatments in patients with nAMD has decreased, leading to a significant vision loss at 6 and 12 months [10-12]. Thus, especially during the COVID-19 pandemic, identifying optimal T\&E treatment intervals, and minimizing clinic visits has become particularly important for patient care and resource planning.

For patients who have a suboptimal response to initial anti-VEGF therapy, switching to an alternative anti-VEGF has been suggested [13]; however, although several single-arm studies have shown the benefits of switching, without a randomized control group, it is not possible to evaluate the effects of switching treatments without the comparison of continuing the original treatment [14]. Outcomes of a hypothetical therapy switch in patients who have a poor initial response but continue anti-VEGF treatment without switching have been evaluated in an analysis of patients from the Comparison of Age-Related Macular Degeneration Treatments Trials (CATT) treated with fixeddose or pro re nata (PRN) ranibizumab or bevacizumab [15], and the Phase 3 HARBOR clinical trial of fixed-dose or PRN ranibizumab [14]. These studies suggested that continuation of initial anti-VEGF therapy will, in many cases, result in gradual improvement or stabilization of the eye $[14,15]$.

The ARIES study was a randomized, openlabel, phase $3 \mathrm{~b} / 4$ study of patients with nAMD and evaluated best-corrected visual acuity (BCVA) in patients randomized at week 16 to either early-start T\&E, with 2-week interval adjustments, or late-start T\&E, with 8-week intervals until week 48 and 2-week interval adjustments thereafter [16]. This post hoc analysis of data from the ARIES study explored functional and anatomic outcomes (BCVA and central retinal thickness [CRT]) in patients with treatment-naïve nAMD who were treated with intravitreal aflibercept (IVT-AFL) for up to 104 weeks, but retrospectively were identified as meeting criteria for a hypothetical switch early during the course of treatment.

For a video summary of our research, please see video 1 in the online/HTML version of the manuscript or follow the digital features link following the abstract.

\section{METHODS}

\section{The ARIES Study}

The study design, ethical approvals, patient inclusion and exclusion criteria, and endpoints of ARIES (ClinicalTrials.gov Identifier: NCT02581891) have been published in full previously [16]. The study was conducted in 
Table 1 Criteria for hypothetical switch

\begin{tabular}{lllll}
\hline $\begin{array}{l}\text { Criterion } \\
\text { Presence of IRF } \\
\text { and/or SRF with } \\
\text { central involvement }\end{array}$ & $\begin{array}{l}\text { Next planned } \\
\text { treatment interval }\end{array}$ & BCVA & Population \\
\hline 1 & Week 8 & Any & Any & Overall population \\
2 & Week 8 & Any & $<70$ and gains $\leq 5$ letters at week 8 & Overall population \\
3 & Week 24 & Any & Any & Overall population \\
4 & Week 24 & Any & $<70$ and gains $\leq 5$ letters at week 24 & Overall population \\
5 & Week 24 & $\leq 8$ weeks at week 24 & Any & $\begin{array}{c}\text { Early-start T\&E } \\
\text { arm }{ }^{\text {a }}\end{array}$ \\
6 & Week 24 & $\leq 8$ weeks at week 24 & $<70$ and gains $\leq 5$ letters at week 24 & $\begin{array}{c}\text { Early-start T\&E } \\
\text { arm }{ }^{\text {a }}\end{array}$ \\
\hline
\end{tabular}

$B C V A$ Best-corrected visual acuity, $I R F$ intraretinal fluid, $S R F$ subretinal fluid, $T \mho E$ treat-and-extend

${ }^{a}$ Criteria 5 and 6 were assessed in the early-start T\&E arm because the treatment interval could not be $>8$ weeks in year 1 in the late-start T\&E arm

accordance with the Declaration of Helsinki and the International Conference on Harmonization guidelines E6: Good Clinical Practice. The protocol and amendment were approved by the independent ethics committee/institutional review board at each site. All patients provided written informed consent to participate in the study.

In brief, patients with treatment-naïve nAMD received three initial monthly doses of $2 \mathrm{mg}$ IVT-AFL, followed by an injection after an 8 -week treatment interval (week 16). At week 16 , patients were randomly assigned to earlystart T\&E (2-week interval adjustments to a maximum interval of 16 weeks) or late-start T\&E (8-week intervals until week 48 then 2-week interval adjustments to a maximum of 16 weeks). The full analysis set included all randomly assigned patients who received IVTAFL and had a BCVA assessment at week 16 and at least one additional BCVA assessment after week 16.

Retinal and lesion characteristics were evaluated using spectral-domain optical coherence tomography (SD-OCT). Images of the study eye and the fellow eye were captured and assessed by study-site personnel specifically trained and certified for this assessment in order to ensure consistency and quality in image acquisition. Images obtained by OCT were evaluated by an independent central reading center. Intraretinal fluid (IRF) was defined as diffuse darkening between the internal limiting membrane and the photoreceptor layer, and subretinal fluid (SRF) was defined as well-defined darkening with a minimal horizontal extension of $100 \mu \mathrm{m}$ between the retinal pigment epithelium and photoreceptor layer. IRF or SRF was considered 'central' if it was present in the central 1000- $\mu \mathrm{m}$ (1-mm)-diameter area.

The anatomic criteria for extending the treatment intervals for the study eye, as assessed by investigators, were the absence of any IRF, absence of new neovascularization or hemorrhage, and SRF not exceeding $50 \mu \mathrm{m}$ in thickness. If extension criteria were not met, treatment intervals were reduced to the last effective interval. The ARIES study stipulated that treatment intervals following randomization should not be $<8$ weeks unless patients 


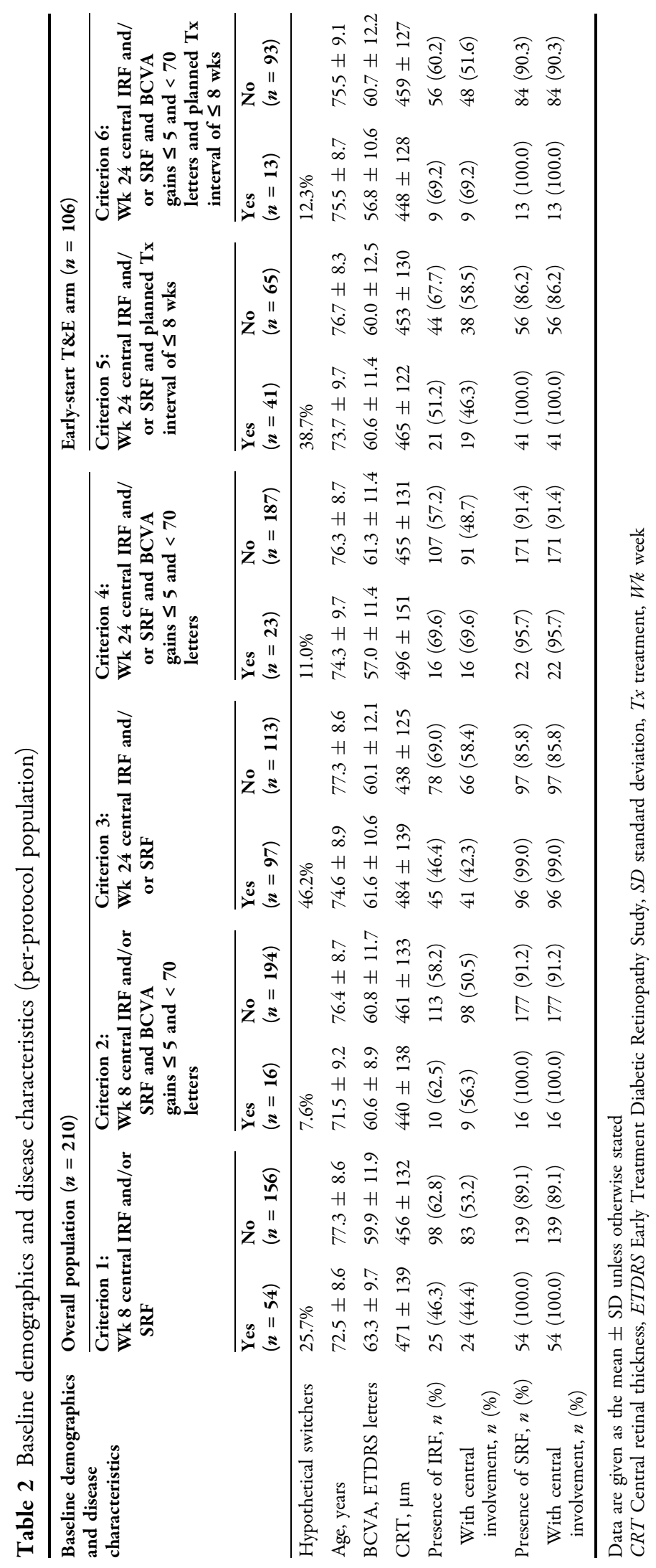


(a)

Hypothetical switchers ( $n=54)$
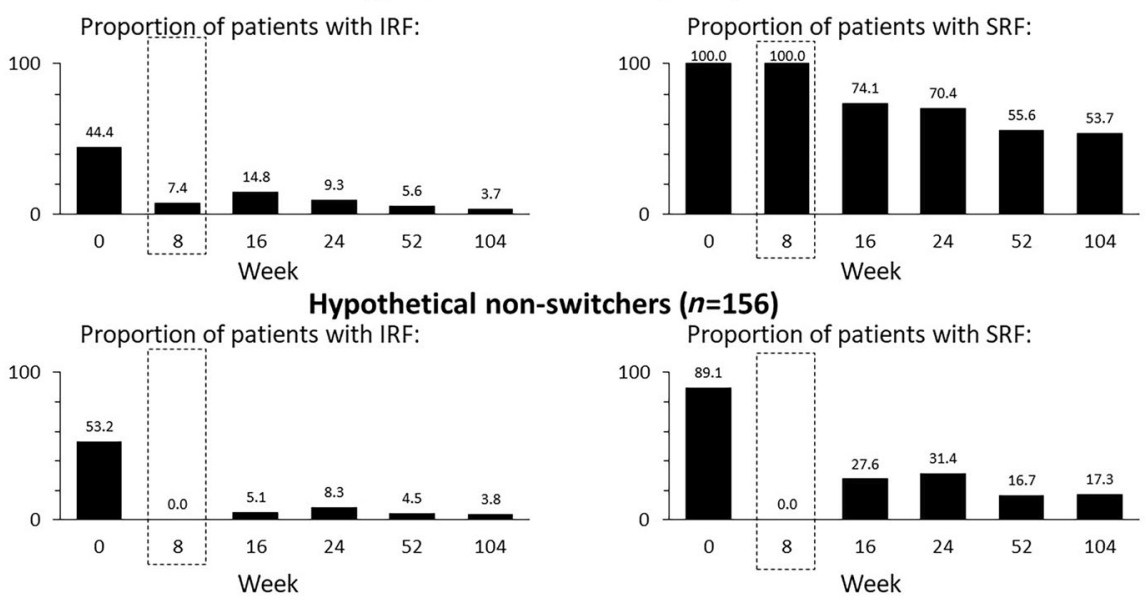

(b)

Hypothetical switchers ( $n=97)$
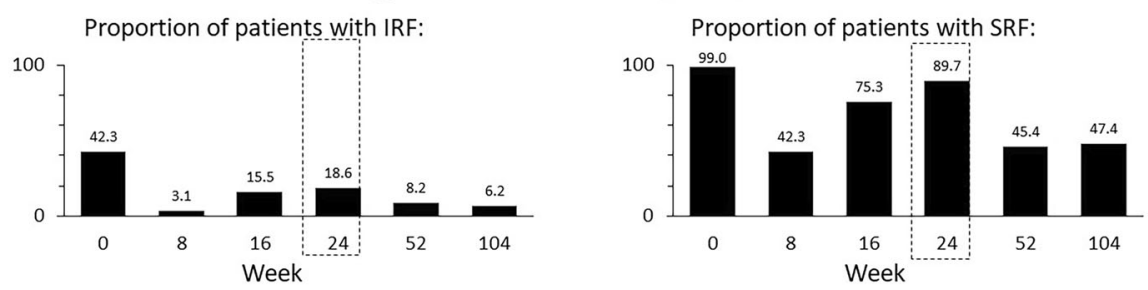
Proportion of patients with IRF:
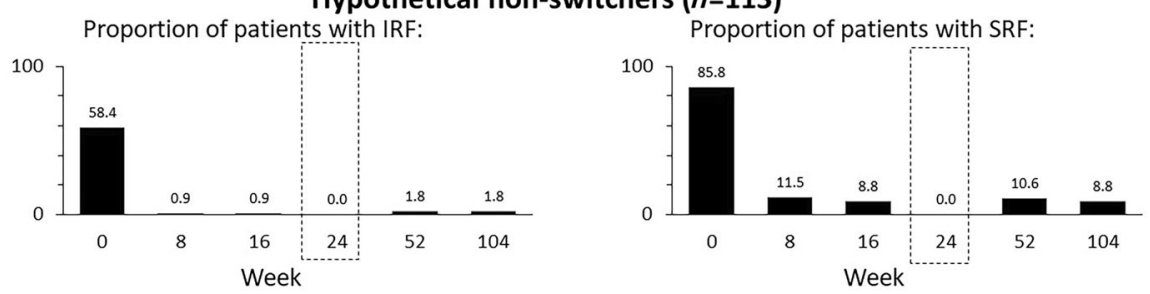

(c)

Hypothetical switchers ( $n=41$ )
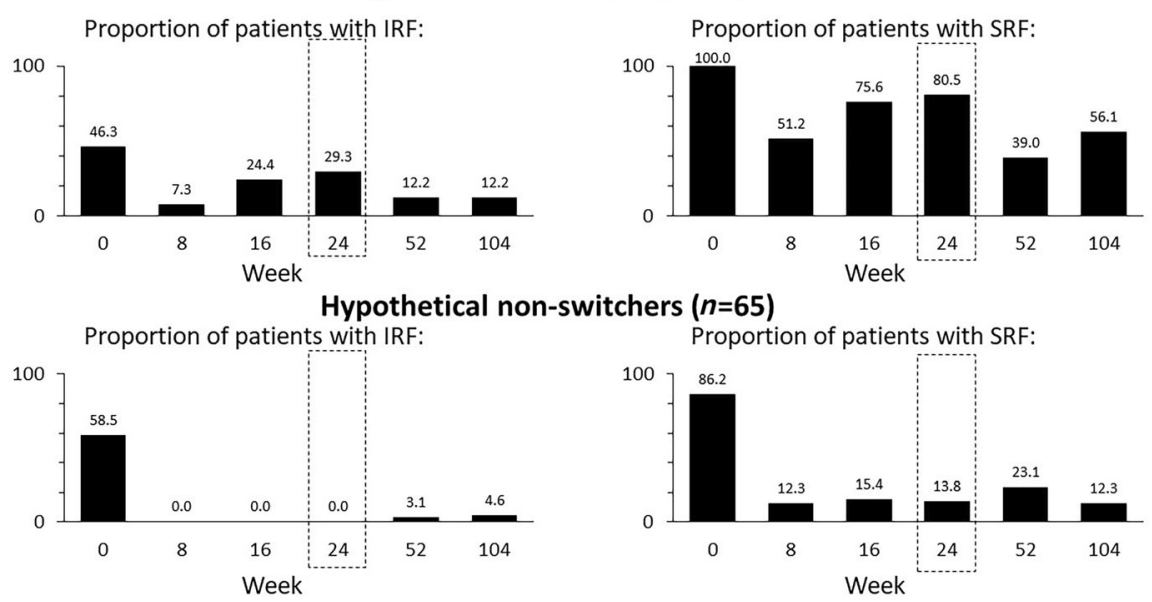
4Fig. 1 Proportion of patients in the per-protocol set with central IRF and central SRF over time, grouped post baseline by hypothetical switch criteria 1, 3, and 5: the presence of central fluid at week 8 (a), week 24 (b), week 24 (c) and next planned treatment interval $\leq 8$ weeks. The dashed box indicates the "index" weeks at which time point hypothetical switch criteria were defined. IRF Intraretinal fluid, $S R F$ subretinal fluid

were considered by the investigator to require injections more frequently. In such cases $(n=62)$, patients were able to continue in the study, but were excluded from the per-protocol population on which the primary endpoint was based.

The primary endpoint of the study was mean change in BCVA (Early Treatment Diabetic Retinopathy Study [ETDRS] letters) from randomization (week 16) to week 104 analyzed in the per-protocol population. Secondary endpoints included mean change in BCVA from baseline to week 52, from baseline to week 104 and from week 16 to week 52, and mean change in CRT from baseline to weeks 52 and 104 and from week 16 to weeks 52 and 104. Other secondary and exploratory efficacy endpoints have been previously published.

\section{Post Hoc Analyses}

These post hoc analyses were conducted on the per-protocol population $(n=210)$ and the full analysis set $(n=269)$. To model real-life switch situations, patients were categorized according to six different criteria (Table 1) based on the presence of either IRF with central involvement or SRF with central involvement at week 8 or 24 , whether or not the next planned treatment interval was $\leq 8$ weeks, and BCVA.

BCVA and CRT from baseline and the hypothetical switch timepoint to weeks 52 and 104 were described, using a last observation carried forward approach for missing values. Due to the post hoc nature of the analysis, data were reported descriptively, and no formal statistical comparisons were conducted.

\section{RESULTS}

In the ARIES study, 271 patients were randomly assigned, 269 were included in the full analysis set, and 236 completed the 104-week study. Of these, 210 patients were included in the perprotocol population. Baseline demographics and disease characteristics in the retrospectively defined, post-baseline groupings are shown in Table 2, with no obvious differences in baseline characteristics between groups.

The proportions of patients considered to have a suboptimal initial response to IVT-AFL varied widely depending on the criteria used (7.6-46.2\%). The application of criteria 2,4 , and 6 , which included an additional BCVA element within the definition, resulted in few patients considered to have a suboptimal initial response to IVT-AFL $(n=13-23)$ and limited meaningful comparison of outcomes with the non-switch group. Analyses therefore focused on criteria excluding BCVA (criteria 1, 3, and 5).

Reasons for discontinuations have been reported previously [16]. Discontinuation rates by week 104 showed no clear trends in patients who did and did not meet hypothetical switch criteria $(3.4 \%$ and $6.5 \%$ based on criterion 1 ; $7.2 \%$ and $6.2 \%$ based on criterion 3 ; and $7.3 \%$ and $4.6 \%$ based on criterion 5). All hypothetical switch criteria included the presence of either IRF or SRF with central involvement. Given that extension criteria included the absence of IRF and SRF not exceeding $50 \mu \mathrm{m}$ in thickness, actual and planned treatment intervals were numerically longer, and the number of injections received by weeks 52 and 104 were numerically higher in the patients who met the hypothetical switch criteria (Electronic Supplementary Material [ESM] Table 1).

The proportion of patients with central IRF and SRF over time in hypothetical switchers and patients who did not meet switch criteria are shown in Fig. 1. Hypothetical switch criteria were largely met due to the presence of central SRF rather than IRF. According to criterion 1, central IRF was present in $7.4 \%$ of hypothetical switchers and central SRF was present in 100\% of hypothetical switchers at week 8 . According to criterion 3, central IRF was present in $18.6 \%$ 
(a)

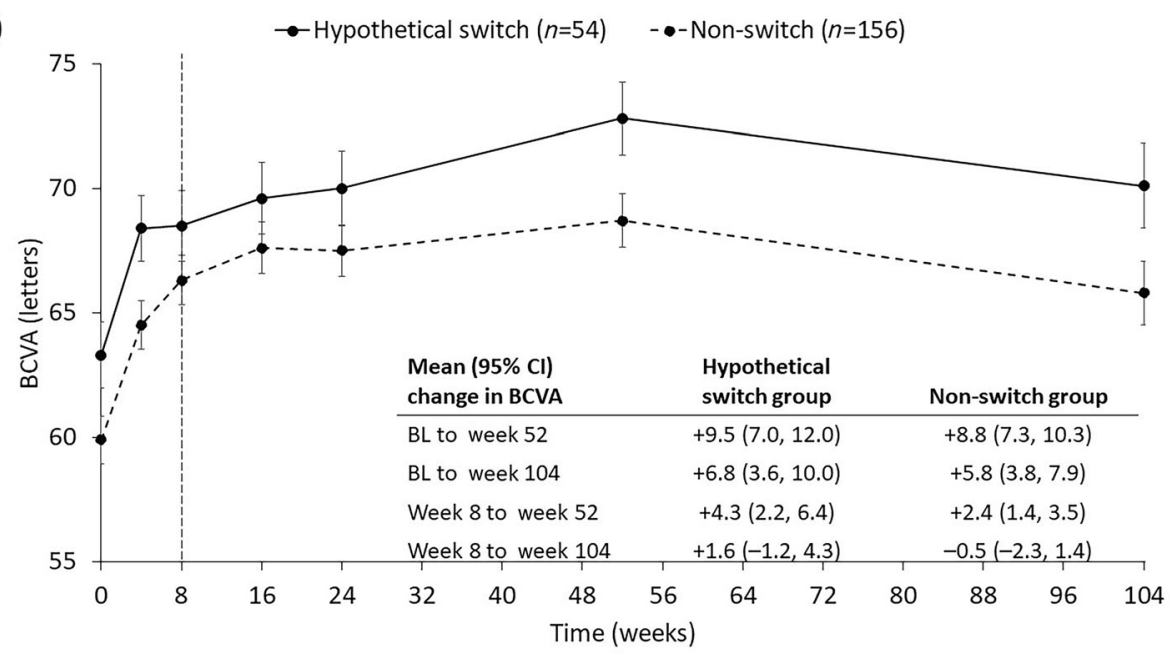

(b)

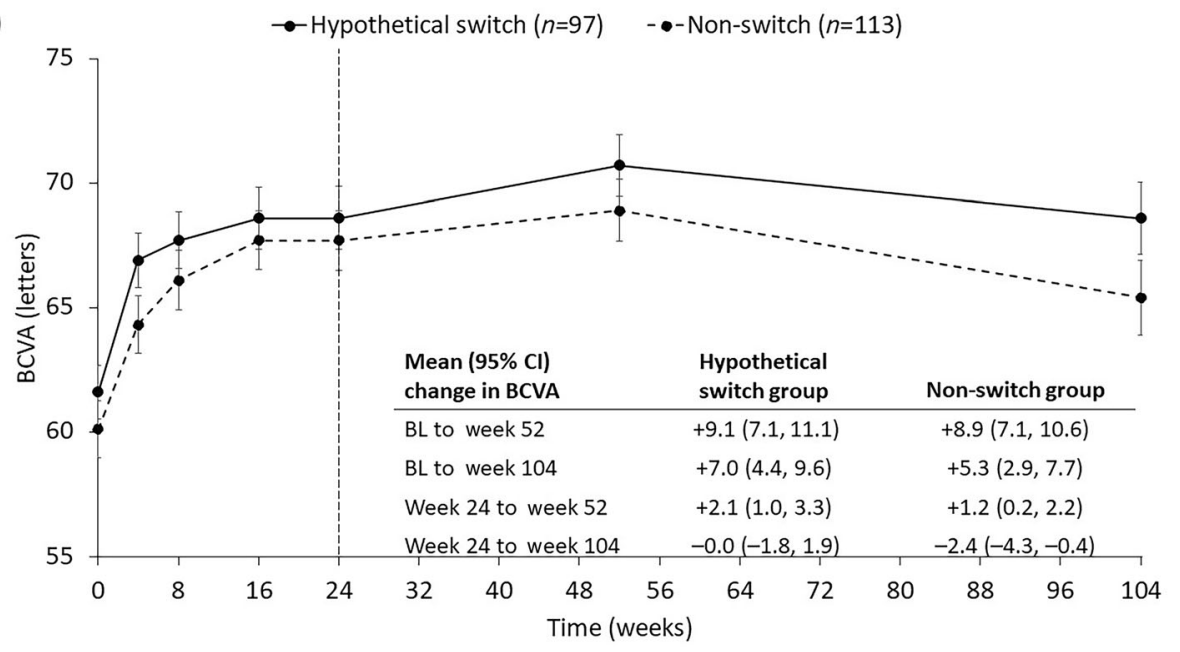

(c)

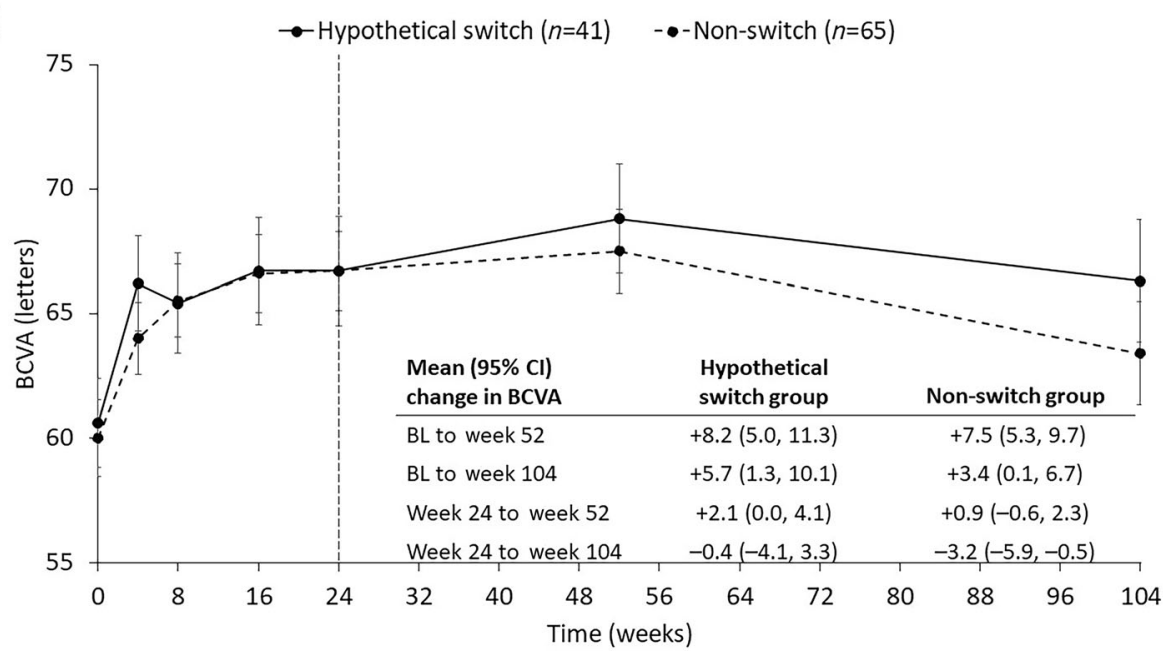


4Fig. 2 Mean ( \pm standard error of the mean [SEM]) absolute BCVA values over time in patients in the perprotocol population grouped post-baseline by hypothetical switch criteria 1,3 , and 5: the presence of central fluid at week 8 (a), week $24(\mathbf{b})$, and week 24 (c) and next planned treatment interval $\leq 8$ weeks. The dashed line indicates the "index" weeks at which point hypothetical switch criteria were defined. $B C V A$ Best-corrected visual acuity, $B L$ baseline, $C I$ confidence interval

of hypothetical switchers and central SRF was present in $89.7 \%$ of hypothetical switchers at week 24. According to criterion 5, central IRF was present in $29.3 \%$ of hypothetical switchers and central SRF was present in $80.5 \%$ of hypothetical switchers at week 24 . There were nine patients in the early T\&E arm in whom central SRF was present at week 24, but the treatment interval was not $\leq 8$ weeks; hence, the proportion of patients with SRF in the hypothetical non-switch group according to criterion 5 was $13.8 \%$.

In patients meeting the hypothetical switch criteria, the proportions of patients with IRF with central involvement were numerically smaller at weeks 52 and 104 than in the "index week" (the time point at which hypothetical switch criteria were met); the same was true for the proportions of patients with SRF with central involvement.

\section{Functional Outcomes}

Absolute BCVA from baseline to week 104 for the per-protocol population is shown in Fig. 2. Irrespective of criteria used, mean BCVA was not worse in those patients who were identified for a hypothetical switch as having an initially suboptimal response to IVT-AFL. According to the presence of either IRF or SRF with central involvement at week 8, mean changes in BCVA from baseline in patients who met criterion 1 and those who did not were +9.5 and +8.8 letters, respectively, at week 52 and +6.8 and +5.8 letters, respectively, at week 104 (Fig. 2a). Mean changes in BCVA from baseline in patients who met criterion 3 and those who did not were +9.1 and +8.9 , respectively, at week 52 and +7.0 and 5.3, respectively, at week 104 (Fig. 2b). Mean changes in BCVA from baseline in patients who met criterion 5 and those who did not were +8.2 and +7.5 letters, respectively, at week 52 and +5.7 and +3.4 letters, respectively, at week 104 (Fig. 2c).

Similar observations were seen in the full analysis set, which included a minority of patients who were treated with IVT-AFL with an interval $<8$ weeks at least once during the study, who were excluded from the per-protocol population (ESM Fig. 1).

\section{Anatomic Outcomes}

Absolute CRT from baseline to week 104 is shown in Fig. 3. Differences between the two groups are shown to be largely driven by the hypothetical switch criteria, all of which included the presence of central fluid.

According to the presence of either IRF or SRF with central involvement at week 8 , mean changes in CRT from baseline in patients who met criterion 1 and those who did not were, respectively, -146 and $-173 \mu \mathrm{m}$ at week 52 , and -141 and $-167 \mu \mathrm{m}$ at week 104 (Fig. 3a). Mean changes in CRT from baseline in patients who met criterion 3 and those who did not were, respectively, -161 and $-171 \mu \mathrm{m}$ at week 52 and -149 and $-169 \mu \mathrm{m}$ at week 104 (Fig. 3b). Mean changes in CRT from baseline in patients who met criterion 5 and those who did not were, respectively, -152 and $-173 \mu \mathrm{m}$ at week 52 and -138 and $-176 \mu \mathrm{m}$ at week 104 (Fig. 3c).

Similar observations were seen in the full analysis set (ESM Fig. 2).

\section{DISCUSSION}

In this post hoc analysis of ARIES, BCVA did not decline, but rather was maintained or numerically increased, during continued IVT-AFL treatment using proactive individualized T\&E regimens (either after three initial monthly injections or after 1 year of dosing every 8 weeks) despite patients meeting hypothetical switch criteria related to the presence of central IRF or SRF. These results were observed 

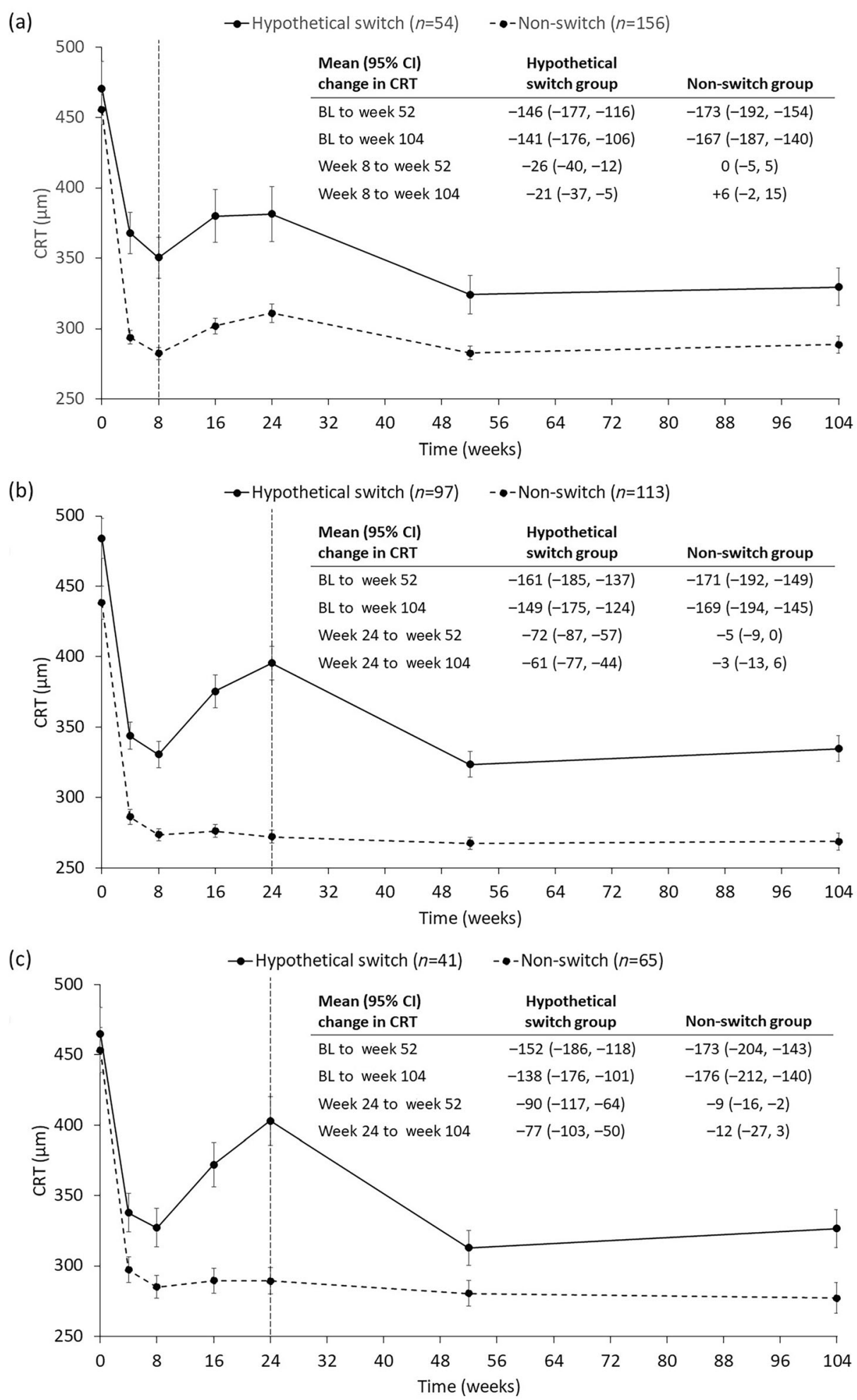
4Fig. 3 Mean ( \pm SEM) absolute CRT values over time in patients in the per-protocol population grouped post baseline by hypothetical switch criteria 1, 3, and 5: the presence of central fluid at week 8 (a), week 24 (b), and week 24 (c) and next planned treatment interval $\leq 8$ weeks. The dashed line indicates the "index" weeks at which point hypothetical switch criteria were defined. $C R T$ Central retinal thickness

irrespective of which of the three different criteria (fluid at week 8 , fluid at week 24 , or fluid at week 24 in addition to a next planned treatment interval $<8$ weeks) were used to indicate a potential switch from IVT-AFL to another antiVEGF agent.

The presence of central SRF, rather than central IRF, was the most common parameter leading to hypothetical switch criteria being met at both week 8 and week 24 . We did not define hypothetical switch criteria according to the presence of central IRF only since few guidelines distinguish between the types of fluid when providing recommendations, with the same retreatment approach generally recommended regardless of the type and location of fluid observed [17]. This is interesting given that studies have shown what appears to be an important difference in the relationship between BCVA and either IRF or SRF [18]. Indeed, the presence of SRF in the central macula was associated with better BCVA in patients with nAMD [19], and the presence of SRF has been proposed as an indicator of a more benign form of nAMD [20].

Anti-VEGF agents have been the gold standard for the treatment of nAMD; however, differences in individual responses are seen, and fluctuations in BCVA and CRT values, which can be relatively inconsequential when averaged across a population, can be more concerning on an individual level [8]. A suboptimal response to anti-VEGF therapy may result from misdiagnosis of the underlying condition and, particularly, insufficient administration of injections [13]. However, there are considerable variations in the use of anti-VEGF agents in nAMD in practice, and how to define suboptimal responses and manage such patients remains a challenge clinically.
One approach to defining responses to antiVEGF therapy was proposed by a UK consensus panel [21]. The panel proposed that an optimal response comprises resolution of fluid (IRF, SRF, and retinal thickening) and/or improvement of $>5$ letters, with poor response defined as $<25 \%$ reduction in CRT and/or persistence of, or new, SRF, IRF, intraretinal cysts, and a change in BCVA of $<4$ letters [21]. According to the authors, the response is best defined 1 month after the last initiation dose (end of month 3 in nAMD). The assumption is that, if treatment with one anti-VEGF agent fails to achieve the desired success by month 4, further treatment with that same anti-VEGF agent is futile. However, the findings of the ARIES analysis are contrary to that assumption, and consistent with similar analyses undertaken based on data from the HARBOR study and CATT [14, 15].

In the HARBOR analysis, all of the following criteria were required for patients to meet criteria for hypothetical switch: BCVA of 20/40 with gain of $\leq 5$ letters, and either IRF or SRF, and central foveal thickness equal to or greater than central subfield thickness at month 3 or month 6 . Based on these criteria, $<5 \%$ of patients met hypothetical switch criteria at months 3 and 6 [14]. Patients had a mean letter gain of 1.8-2.7 from switch to month 24 , with significant reductions $(-111$ and $-136 \mu \mathrm{m}$, depending on criteria) in central foveal thickness [14]. Similarly, in the CATT analysis, hypothetical switch criteria required BCVA $20 / 40$ or worse, and gain $\leq 1$ letters and persistent CRT at month 3 or month 6 [15]. Based on these criteria, $7 \%$ and $11 \%$ of patients met hypothetical switch criteria at months 3 and 6 , respectively. Patients had a 3- to 5-letter gain and $40-70 \mu \mathrm{m}$ reduction in CRT after switch criteria were met [15].

The data reported here for ARIES focus on the population of patients defined by more stringent switch criteria than those used in the HARBOR or CATT analyses, and exclude BCVA components from the definitions. In ARIES, very few patients $(8-12 \%)$ fulfilled switch criteria when a BCVA of $<70$ letters, with gains $\leq 5$ letters, was included in the criteria to define hypothetical switch. Therefore, we concentrated our analyses on those definitions 
focusing solely on the presence of fluid with or without a next treatment interval $\leq 8$ weeks. This is largely aligned with clinical practice, where there is a shift from functional to morphological outcomes to drive treatment decisions. Using the defined criteria in this analysis (excluding BCVA), $26-46 \%$ of patients met criteria for a hypothetical switch. Due to the ARIES study protocol and assessment time points, it was not possible to define the switching time point at month 3 and month 6 , as was done with the CATT and HARBOR analyses; the ARIES switch time points were 8 and 24 weeks. Thus, direct comparisons between the ARIES analysis and the HARBOR and CATT analyses should not be made.

We should acknowledge that this was a post hoc analysis, which was not designed to evaluate the effects of continuing the original antiVEGF agent in patients who have a suboptimal initial response to IVT-AFL. Strengths include presentation of data in both patients who met and did not meet hypothetical switch criteria. Although comparisons between the hypothetical switch and non-switch groups should not be made, due to the retrospective allocation of patients to these groups based on post-baseline outcomes, it is interesting to see numerically better BCVA outcomes in patients meeting switch criteria. This is likely related to the fact that most patients met switch criteria due to the presence of SRF (in the absence of IRF). Numerically greater improvements in BCVA in patients with stable, residual SRF have also been observed in post hoc analyses of other studies of patients with nAMD [22-25].

\section{CONCLUSIONS}

In summary, in patients in the ARIES study with nAMD who met criteria for a hypothetical switch of anti-VEGF treatment, mean initial improvements in BCVA were maintained or even numerically improved with continued proactive, individualized T\&E IVT-AFL therapy. Based on our findings, there is limited rationale for switching treatment-naïve nAMD patients from IVT-AFL during the first half year of therapy because, with continuous proactive treatment, comparable visual gains can be achieved to those who initially respond well on a T\&E regimen over a 2-year timeframe. Since the ARIES study follow-up was 104 weeks, further studies are needed to determine the relevance of our findings over a longer period of time.

\section{ACKNOWLEDGEMENTS}

The authors thank all the patients and investigators who participated in the ARIES study.

Funding. The ARIES study was sponsored by Bayer AG, Germany. Bayer Consumer Care AG funded the post hoc analysis and is also responsible for funding the journal's Rapid Service and Open Access Fees.

Medical Writing, Editorial and Other Assistance. Medical writing and editorial support for the preparation of this manuscript, under the direction of the authors, was provided by Sarah Feeny, BMedSci, ApotheCom (London) and was funded by Bayer Consumer Care AG, Basel, Switzerland, in accordance with Good Publication Practice (GPP3) guidance (Ann Intern Med 2015;163:461-464). The authors would also like to acknowledge the contributions of Nader Botros (Bayer AG), for valuable insights during the initial development of the concept and at several stages throughout the analysis.

Prior Presentation. Data were presented in part at the European Society of Retina Specialists (EURETINA) 2021 Virtual meeting, 9-12 September, 2021 in the Prize Paper category.

Authorship. All named authors meet the International Committee of Medical Journal Editors (ICMJE) criteria for authorship for this article, take responsibility for the integrity of the work as a whole, and have given their approval for this version to be published.

Author Contributions. All authors developed the hypothesis and overall concept. Tobias Machewitz conducted the statistical analysis. 
All authors were involved in the interpretation of the results, and the review and development of the manuscript. All authors have provided final approval of the manuscript to be published and agree to be accountable for all aspects of the work by ensuring that questions related to the accuracy or integrity of any part of the work are appropriately investigated and resolved.

Compliance with Ethics Guidelines. The ARIES study was conducted in accordance with the Declaration of Helsinki and the International Conference on Harmonization guidelines E6: Good Clinical Practice. The protocol and amendment were approved by the independent ethics committee/institutional review board at each site. All patients provided written informed consent to participate in the study.

Disclosures. Cengiz Türksever: Consultant/ advisor for Allergan, Bayer, and Novartis. Gábor Márk Somfai: Consultant/advisor for Allergan, Bayer, Novartis, and Roche. Susanne Oesch: Employee of Bayer (Schweiz) AG. Tobias Machewitz: Employee of Bayer AG. Pascal W. Hasler: Consultant/advisor for Roche; speaker for Bayer, Novartis, and Topcon. Sandrine Zweifel: Consultant/advisor for Bayer Healthcare Pharmaceuticals, Novartis Pharmaceuticals Corp., Roche, and Carl Zeiss Meditec AG; grant support from Novartis Pharmaceuticals Corp. and Bayer Healthcare Pharmaceuticals.

Data Availability. Availability of the data underlying this publication will be determined later according to Bayer's commitment to the EFPIA/PhRMA "Principles for responsible clinical trial data sharing." This pertains to scope, time point, and process of data access. As such, Bayer commits to sharing upon request from qualified scientific and medical researchers patient-level clinical trial data, study-level clinical trial data, and protocols from clinical trials in patients for medicines and indications approved in the USA and EU as necessary for conducting legitimate research. This applies to data on new medicines and indications that have been approved by the EU and US regulatory agencies on or after 01 January 2014. Interested researchers can use https://www. clinicalstudydatarequest.com to request access to anonymized patient-level data and supporting documents from clinical studies to conduct further research that can help advance medical science or improve patient care. Information on the Bayer criteria for listing studies and other relevant information is provided in the study sponsors section of the portal. Data access will be granted to anonymized patient-level data, protocols, and clinical study reports after approval by an independent scientific review panel. Bayer is not involved in the decisions made by the independent review panel. Bayer will take all necessary measures to ensure that patient privacy is safeguarded.

Open Access. This article is licensed under a Creative Commons Attribution-NonCommercial 4.0 International License, which permits any non-commercial use, sharing, adaptation, distribution and reproduction in any medium or format, as long as you give appropriate credit to the original author(s) and the source, provide a link to the Creative Commons licence, and indicate if changes were made. The images or other third party material in this article are included in the article's Creative Commons licence, unless indicated otherwise in a credit line to the material. If material is not included in the article's Creative Commons licence and your intended use is not permitted by statutory regulation or exceeds the permitted use, you will need to obtain permission directly from the copyright holder. To view a copy of this licence, visit http://creativecommons.org/licenses/bync/4.0/.

\section{REFERENCES}

1. Taylor DJ, Hobby AE, Binns AM, Crabb DP. How does age-related macular degeneration affect realworld visual ability and quality of life? A systematic review. BMJ Open. 2016;6:e011504.

2. Assi L, Chamseddine F, Ibrahim P, et al. A global assessment of eye health and quality of life: a systematic review of systematic reviews. JAMA Ophthalmol. 2021;139:526-41. 
3. Moshfeghi AA, Lanitis T, Kropat G, et al. Social cost of blindness due to AMD and diabetic retinopathy in the United States in 2020. Ophthalmic Surg Lasers Imaging Retin. 2020;51:S6-14.

4. Wong WL, Su X, Li X, et al. Global prevalence of age-related macular degeneration and disease burden projection for 2020 and 2040: a systematic review and meta-analysis. Lancet Glob Health. 2014;2:e106-16.

5. Rein DB, Wittenborn JS, Zhang X, et al. Forecasting age-related macular degeneration through the year 2050: the potential impact of new treatments. Arch Ophthalmol. 2009;127:533-40.

6. Colijn JM, Buitendijk GHS, Prokofyeva E, et al. Prevalence of age-related macular degeneration in Europe: the past and the future. Ophthalmology. 2017;124:1753-63.

7. Novartis. LUCENTIS ${ }^{\circledR}$ (ranibizumab injection) for intravitreal injection [prescribing information]. https://www.accessdata.fda.gov/drugsatfda_docs/ label/2014/125156s105lbl.pdf. 2018. Accessed Jan 2022.

8. Schmidt-Erfurth U, Chong V, Loewenstein A, et al. Guidelines for the management of neovascular agerelated macular degeneration by the European Society of Retina Specialists (EURETINA). $\mathrm{Br} \mathrm{J}$ Ophthalmol. 2014;98:1144-67.

9. Gale RP, Mahmood S, Devonport H, et al. Action on neovascular age-related macular degeneration (nAMD): recommendations for management and service provision in the UK hospital eye service. Eye (Lond). 2019;33:1-21.

10. Arruabarrena $\mathrm{C}$, Toro MD, Onen M, et al. Impact on visual acuity in neovascular age related macular degeneration (nAMD) in Europe due to COVID-19 pandemic lockdown. J Clin Med. 2021;10:3281.

11. Stattin M, Ahmed D, Graf A, et al. The effect of treatment discontinuation during the COVID-19 pandemic on visual acuity in exudative neovascular age-related macular degeneration: 1-year results. Ophthalmol Ther. 2021;10:1-11.

12. Bajka A, Wiest MRJ, Hamann T, Toro MD, Zweifel SA. Assessment of patients' confidence regarding a new triage concept in a medical retina clinic during the first COVID-19 outbreak. Int J Environ Res Public Health. 2021;18:5846.

13. Wong DT, Lambrou GN, Loewenstein A, et al. Suspending treatment of neovascular age-related macular degeneration in cases of futility. Retina. 2020;40:1010-20.
14. Zarbin M, Tsuboi M, Hill LF, Stoilov I. Simulating an anti-vascular endothelial growth factor switch in neovascular age-related macular degeneration: a HARBOR subanalysis. Ophthalmology. 2019;126: 849-55.

15. Ferris FL 3rd, Maguire MG, Glassman AR, Ying GS, Martin DF. Evaluating effects of switching antivascular endothelial growth factor drugs for agerelated macular degeneration and diabetic macular edema. JAMA Ophthalmol. 2017;135:145-9.

16. Mitchell P, Holz FG, Hykin P, et al. Efficacy and safety of intravitreal aflibercept using a treat-andextend regimen for neovascular age-related macular degeneration: the ARIES study. Retina. 2021;41: 1911-20.

17. Kodjikian L, Parravano M, Clemens A, et al. Fluid as a critical biomarker in neovascular age-related macular degeneration management: literature review and consensus recommendations. Eye. 2021;35:2119-35.

18. Chaudhary V, Matonti F, Zarranz-Ventura J, Stewart MW. The impact of fluid compartments on functional outcomes for patients with neovascular agerelated macular degeneration: a systematic literature review. Retina. 2021. https://doi.org/10.1097/ IAE.0000000000003283.

19. Zarbin MA, Hill L, Maunz A, Gliem M, Stoilov I. Anti-VEGF-resistant subretinal fluid is associated with better vision and reduced risk of macular atrophy. Br J Ophthalmol. 2021. https://doi.org/10. 1136/bjophthalmol-2020-318688.

20. Siedlecki J, Fischer C, Schworm B, et al. Impact of sub-retinal fluid on the long-term incidence of macular atrophy in neovascular age-related macular degeneration under treat and extend anti-vascular endothelial growth factor inhibitors. Sci Rep. 2020;10:8036.

21. Amoaku WM, Chakravarthy U, Gale R, et al. Defining response to anti-VEGF therapies in neovascular AMD. Eye (Lond). 2015;29:721-31.

22. Sharma S, Toth CA, Daniel E, et al. Macular morphology and visual acuity in the second year of the comparison of age-related macular degeneration treatments trials. Ophthalmology. 2016;123: $865-75$.

23. Regillo CD, Busbee BG, Ho AC, Ding B, Haskova Z. Baseline predictors of 12-month treatment response to ranibizumab in patients with wet age-related macular degeneration. Am J Ophthalmol. 2015;160:1014-23.

24. Waldstein SM, Simader C, Staurenghi G, et al. Morphology and visual acuity in aflibercept and 
ranibizumab therapy for neovascular age-related macular degeneration in the VIEW trials. Ophthalmology. 2016;123:1521-9.

25. Guymer RH, Markey CM, McAllister IL, et al. Tolerating subretinal fluid in neovascular age-related macular degeneration treated with ranibizumab using a treat-and-extend regimen: FLUID study 24-month results. Ophthalmology. 2019;126: 723-34. 